\title{
MIS-C: how do patients fare 6 months on?
}
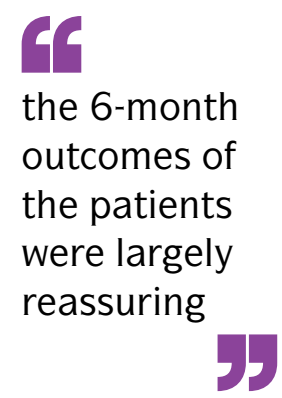

Multisystem inflammatory syndrome in children (MIS-C; also known as PIMS-TS) is a rare post-infectious manifestation of SARS-CoV-2 infection in children. Most studies of MIS-C have been limited to the acute phase of illness, whereas less is known about the medium and long-term outcomes and sequelae of this condition. Newly published results in The Lancet Child \& Adolescent Health provide insight into the 6-month outcomes of MIS-C.

The retrospective cohort consisted of 46 children who were admitted to Great Ormond Street Hospital (a tertiary paediatric hospital in the UK) between 4 April and 1 September 2020, and followed up 6 weeks and 6 months after discharge.

"As this was a completely new disease, we did not know what the consequences of this disease would be. Therefore, we set up a multidisciplinary team clinic, which involved all the professionals who had been involved in the patients' care as inpatients, so we could provide comprehensive holistic follow-up for them as outpatients," explains Karyn Moshal, corresponding author on the study.

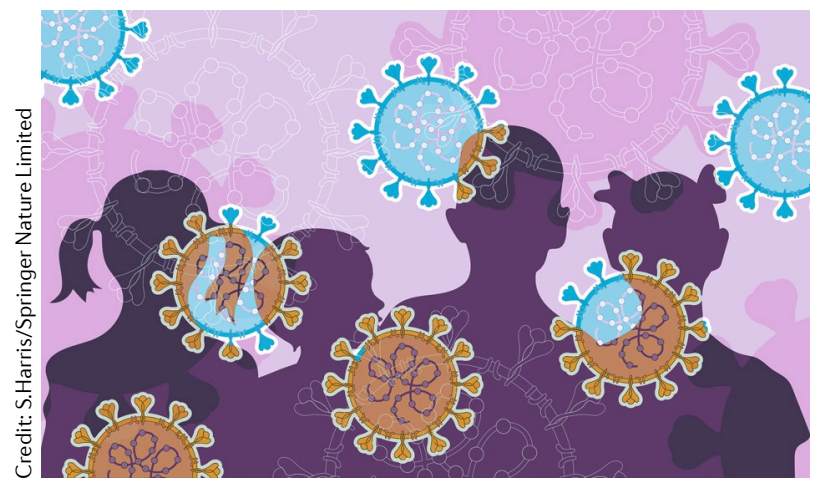

"In seeing the patients as a group, we could learn from each other, and in meticulously documenting the course of each patient, we could pick up patterns of sequelae across the group and best determine the interventions they required."

"Not only was this new disease process a black box for clinicians but also for parents and children. As MIS-C generally affects otherwise well children who have had no or very little contact with healthcare services prior, this created lots of anxiety for parents and children alike," says Justin Penner, first author on the study. "Our approach allowed us to alleviate some of these anxieties whilst providing appropriate specialist psychological support to children and their families throughout. It has also allowed us to recognise and address important psychological and psychosocial trends such as stigma, shame, and medical vulnerability and discuss important issues such as vaccine hesitancy in our patient cohort."

The 6-month outcomes of the patients were largely reassuring. Systemic inflammation had resolved in all but one patient. Similarly, many of the severe organ-specific manifestations seen at 6 weeks, including various cardiac, gastrointestinal, renal, haematologic and otolaryngologic symptoms, had mostly resolved by 6 months. Nevertheless, some issues persisted in a subset of patients, including muscle weakness and fatigue, subtle neurological findings and psychological issues (such as emotional lability and anxiety).

As acknowledged by the authors, the retrospective design of the study had a number of limitations.
"Of most important note was that there were no controls and much of what was observed for the central nervous system and muscle findings may be due to severe illness of any sort and the requirement for intensive care management and not specific for MIS-C," says Rae Yeung, a researcher with a strong interest in MIS-C who was not involved in the study. "It is difficult to tease out the contributions from the inflammatory components unique to children with MIS-C from general severe disease requiring ICU admission."

These limitations also applied to the findings about anxiety and emotional lability. "Because there was no comparison group of children with other serious illness requiring intensive care or prolonged hospitalization, it is difficult to know what to make of the psychological impact of the disorder," remarks Anne Rowley, another researcher independent of the study with an interest in MIS-C. "We know that psychological disorders in children are greatly increased in the pandemic era compared with the pre-pandemic era, so the pandemic itself could have had a psychological impact. Because there were no pre-illness data on some assessed parameters it is possible that some impairments were pre-existing."

Nevertheless, the information gained over the course of the study enabled the clinicians to adjust their own practices and highlighted the importance of physical and mental rehabilitation. The researchers plan to continue to provide comprehensive holistic care for this group of patients and monitor them to identify patterns of persistent or new symptoms over time.

Jessica McHugh

ORIGINAL ARTICLE Penner, J. et al. 6-month multidisciplinary follow-up and outcomes of patients with paediatric inflammatory multisystem syndrome (PIMS-TS) at a UK tertiary paediatric hospital: a retrospective cohort study. Lancet Child Adolesc. Health https://doi.org/10.1016/ S2352-4642(21)00138-3 (2021) 